Differential Forms Basis

Functions for Better Conditioned Integral Equations

B. Fasenfest, D. White, M. Stowell, R. Rieben, R. Sharpe, N. Madsen, J. D. Rockway, N. J. Champagne, V. Jandhyala, J. Pingenot

January 20, 2005

2005 IEEE AP-S International Symposium Washington, DC, United States July 3, 2005 through July 8, 2005 
This document was prepared as an account of work sponsored by an agency of the United States Government. Neither the United States Government nor the University of California nor any of their employees, makes any warranty, express or implied, or assumes any legal liability or responsibility for the accuracy, completeness, or usefulness of any information, apparatus, product, or process disclosed, or represents that its use would not infringe privately owned rights. Reference herein to any specific commercial product, process, or service by trade name, trademark, manufacturer, or otherwise, does not necessarily constitute or imply its endorsement, recommendation, or favoring by the United States Government or the University of California. The views and opinions of authors expressed herein do not necessarily state or reflect those of the United States Government or the University of California, and shall not be used for advertising or product endorsement purposes. 


\title{
Differential Forms Basis Functions for Better Conditioned Integral Equations
}

\author{
Benjamin J. Fasenfest ${ }^{*}$, Dan White, Mark Stowell, Rob Rieben, \\ Rob Sharpe, Niel Madsen and John D. Rockway \\ Lawrence Livermore National Laboratory, Livermore, CA \\ fasenfest1@,1ln1.gov
}

\author{
Nathan J. Champagne \\ Louisiana Tech University, Ruston, LA
}

Vikram Jandhyala and James Pingenot

University of Washington, Seattle, WA

\section{Introduction}

Differential forms offer a convenient way to classify physical quantities and set up computational problems. By observing the dimensionality and type of derivatives (divergence, curl, gradient) applied to a quantity, an appropriate differential form can be chosen for that quantity. To use these differential forms in a simulation, the forms must be discretized using basis functions. The 0 -form through 2 -form basis functions are formed for surfaces. Twisted 1-form and 2-form bases will be presented in this paper. Twisted 1-form ( $1^{\wedge}$-forms) basis functions $(\Lambda)$ are divergence-conforming edge basis functions with units $\mathrm{m}^{-1}$. They are appropriate for representing vector quantities with continuous normal components, and they belong to the same function space as the commonly used RWG bases [1]. They are used here to formulate the frequency-domain EFIE with Galerkin testing. The 2-form basis functions $(f)$ are scalar basis functions with units $\mathrm{m}^{-2}$ and with no enforced continuity between elements. At lowest order, the 2form basis functions are similar to pulse basis functions. They are used here to formulate an electrostatic integral equation. It should be noted that the derivative of an $n$-form differential form basis function is an $(n+1)$-form, i.e. the derivative of a $1^{\wedge}$-form basis function is a 2-form. Because the basis functions are constructed such that they have spatial units, the spatial units are removed from the degrees of freedom, leading to a better-conditioned system matrix. In this conference paper, we look at the performance of these differential forms and bases by examining the conditioning of matrix systems for electrostatics and the EFIE. The meshes used were refined across the object to consider the behavior of these basis transforms for elements of different sizes.

\section{Bases Transformation and Rules}

Construction of surface differential forms interpolatory basis functions are now presented to complement the volumetric forms previously defined in [2]. The basis functions are constructed using Lagrange interpolatory polynomials on the reference element, and then transformed to the actual element. The transformed bases are not strictly interpolatory due to the units of the basis functions. All surface elements in a physical mesh are topologically equivalent to a reference element defined on the unit element in the reference coordinate system $\left\{\hat{x}_{1}, \hat{x}_{2}\right\}$. We define all mesh elements in this reference coordinate system defined by the accent hat symbol, $\hat{\Omega}=\left\{\hat{x}_{1}, \hat{x}_{2}\right\}$. Now let $\hat{\mathbf{r}}=\left\{\hat{x}_{1}, \hat{x}_{2}\right\}$ denote an arbitrary point in the reference coordinate system, and $\mathbf{r}=\left\{x_{1}, x_{2}, x_{3}\right\}$ denote a point in physical space. There exists a mapping $\Phi$ from the reference element in $\hat{\Omega}$ to the physical element in $\Omega$. This mapping and its Jacobian are defined as

\footnotetext{
${ }^{1}$ This work was performed under the auspices of the U.S. Department of Energy by the University of California Lawrence Livermore National Laboratory under contract No. W-7405-ENG-48, UCRL-CONF-209109
} 


$$
\mathbf{r}=\Phi(\hat{\mathbf{r}}), \mathbf{J}_{i, j}=\frac{\partial x_{j}}{\partial \hat{x}_{i}}
$$

Following the approach in [2] for finite element methods, all basis functions are defined on a reference square element using the Lagrange polynomial of degree $p$,

$$
L_{i}^{p}(x ; X)=\prod_{\substack{j=0 \\ j \neq i}}^{p} \frac{\left(x-X_{j}\right)}{\left(X_{i}-X_{j}\right)},
$$

where $X$ is a set of $p+1$ interpolation points, then transformed to the actual element as shown in Table 1. The reference element basis function is presented only for quads, but the transformation rules remain the same for both quads and triangles. The referencespace approach is in contrast to that presented in [3], where the basis functions are formed on the actual element. The approach used here allows the reference space basis functions to be sampled and stored once per integration quadrature point used. This avoids the re-computation of the reference space basis functions for every interaction. Only the appropriate transformations must be recomputed for the actual elements.

\section{1-Forms and Twisted 1-Forms}

The 1-form basis functions $(\mathbf{W})$ correspond to curl-conforming finite element basis functions. Twisted 1-form basis functions $(\Lambda)$ are divergence-conforming edge basis functions. These basis functions are "twisted" in the sense that they are rotated $90^{\circ}$ from the standard 1-form basis functions. They are defined as

$$
\Lambda=\boldsymbol{W} \times \hat{\boldsymbol{n}},
$$

which must hold true on both the reference and actual element. Let $\hat{\mathbf{W}}$ and $\hat{\Lambda}$ denote a 1 -form and $1^{\wedge}$-form basis function on the reference element. We break both $\hat{\mathbf{W}}$ and $\hat{\Lambda}$ into two mutually disjoint subsets, consisting of the basis functions associated with an edge $\left(\hat{\mathbf{W}}_{e}, \hat{\Lambda}_{e}\right)$ and shared among elements and the basis functions associated with the face $\left(\hat{\mathbf{W}}_{f}, \hat{\Lambda}_{f}\right)$, which are unique to a single element. The 1 -forms and $1^{\wedge}$-form basis functions for the reference quadrilaterals are shown in Table 1 . The transformations to physical space of these forms are listed in Table 2.

\section{2-forms}

The 2-form basis functions $(\mathbf{F})$ are scalar basis functions with no enforced continuity between elements. The 2 -form basis functions on the reference element are defined as

$$
\hat{\mathbf{F}}=\left\{\begin{array}{l}
L_{i}^{p-1}\left(\hat{x}_{1}\right) L_{j}^{p}\left(\hat{x}_{2}\right) \hat{\mathbf{x}}_{\mathbf{1}} \\
L_{i}^{p-1}\left(\hat{x}_{2}\right) L_{j}^{p}\left(\hat{x}_{1}\right) \hat{\mathbf{x}}_{\mathbf{2}}
\end{array}\right\} \quad i, j=0 \ldots p-1 .
$$

The transformation to physical space is given by

Table 1. The 1-forms for a quadrilateral.

$$
\begin{aligned}
& \text { 1-Forms } \\
& \hat{\mathbf{W}}_{e}=\left\{\begin{array}{l}
L_{i}^{p-1}\left(\hat{x}_{1}\right) L_{j}^{p}\left(\hat{x}_{2}\right) \hat{\mathbf{x}}_{1} \\
L_{i}^{p-1}\left(\hat{x}_{2}\right) L_{j}^{p}\left(\hat{x}_{1}\right) \hat{\mathbf{x}}_{2}
\end{array}\right\}, \begin{array}{c}
i=0, p ; \\
j=0 \ldots p-1
\end{array} \quad \hat{\Lambda}_{e}=\left\{\begin{array}{c}
-L_{i}^{p-1}\left(\hat{x}_{1}\right) L_{j}^{p}\left(\hat{x}_{2}\right) \hat{\mathbf{x}}_{2} \\
L_{i}^{p}\left(\hat{x}_{1}\right) L_{j}^{p-1}\left(\hat{x}_{2}\right) \hat{\mathbf{x}}_{1}
\end{array}\right\}, \begin{array}{c}
i=0, p ; \\
j=0 \ldots p-1
\end{array} \\
& \hat{\mathbf{W}}_{f}=\left\{\begin{array}{l}
L_{i}^{p-1}\left(\hat{x}_{1}\right) L_{j}^{p}\left(\hat{x}_{2}\right) \hat{\mathbf{x}}_{1} \\
L_{i}^{p-1}\left(\hat{x}_{2}\right) L_{j}^{p}\left(\hat{x}_{1}\right) \hat{\mathbf{x}}_{2}
\end{array}\right\} i, j=0 \ldots p-1 \hat{\Lambda}_{f}=\left\{\begin{array}{c}
-L_{i}^{p-1}\left(\hat{x}_{1}\right) L_{j}^{p}\left(\hat{x}_{2}\right) \hat{\mathbf{x}}_{2} \\
L_{i}^{p-1}\left(\hat{x}_{2}\right) L_{j}^{p}\left(\hat{x}_{1}\right) \hat{\mathbf{x}}_{1}
\end{array}\right\} i, j=0 \ldots p-1
\end{aligned}
$$


Table 2. The transformations of the 1-forms to physical space.

$$
\begin{aligned}
& \text { 1-Forms } \\
& \mathbf{W}(\mathbf{r})=[J] \hat{\mathbf{W}}(\hat{\mathbf{r}}) \quad\left[m^{-1}\right] \\
& \nabla \times \mathbf{W}(\mathbf{r})=\frac{[J]^{\mathrm{T}}}{\|[J]\|} \hat{\nabla} \times \hat{\mathbf{W}}(\hat{\mathbf{r}}) \quad\left[m^{-2}\right] \quad \nabla \Lambda(\mathbf{r})=\frac{1}{\|J\|} \hat{\nabla} \hat{\Lambda}_{i j}(\hat{\mathbf{r}}) \quad\left[m^{-2}\right] \\
& \mathbf{F}(\mathbf{r})=\frac{1}{\|[J]\|} \hat{\mathbf{F}}(\hat{\mathbf{r}}) \quad\left[m^{-3}\right] \\
& \text { Twisted 1-Forms } \\
& \Lambda(\mathbf{r})=\frac{[J]^{T}}{\|J\|} \hat{\Lambda}(\hat{\mathbf{r}}) \quad\left[m^{-1}\right]
\end{aligned}
$$

\section{Results}

The 2-forms were used to represent surface charge on conducting bodies. An integral equation for voltage in terms of charge was formulated using Galerkin testing. This method was compared with a formulation using centroid collocation and unitary pulse basis functions, a common formulation for electrostatics. The sample problem of a unit cube in free space at $1 \mathrm{~V}$ potential was used to test conditioning. The rectangular patches were clustered closer to the edges of the cube so that the ratio of the size of any patch to its neighbor was 1.4. This allowed for better resolution of edge affects and created a range of element sizes, as would be found in a real mesh. The conditioning of the system matrix for the differential forms basis functions is better than that for the unitary pulse basis functions as the mesh size increases, as shown in Figure 1.

The EFIE was implemented using the differential $1^{\wedge}$-forms basis functions [2]. The conditioning of the system matrix was compared against that of EIGER [4], a well-tested frequency-domain code that uses standard RWG basis functions. Mesh refinements of the NASA almond (Figure 2) were simulated in both codes. The condition numbers for EIGER and the differential forms basis functions are compared in Figure 3.

\section{Conclusions}

Discretized differential-forms basis functions were formulated and implemented for surface integral equation problems. Making the degrees of freedom scale invariant with element size leads to better conditioning of the system matrix for both electrostatic and frequency-domain scattering problems on meshes with a large variation in patch size.

\section{References}

[1] S. Rao, D. Wilton, and A. Glisson, "Electromagnetic Scattering by Surfaces of Arbitrary Shape," IEEE Trans. Antennas and Propagation, Vol. 30, no. 3, pp409-418, 1982.

[2] R. Rieben, D. White and G. Rodrigue, "Improved Conditioning of Finite Element Matrices using New High Order Interpolatory Bases," IEEE Trans. Antennas and Propagation, Vol. 52, no. 10, pp. 2675-2683, October 2004.

[3] R.Graglia, D. Wilton, and A. Peterson, "Higher order interpolatory vector bases for computational electromagnetics," IEEE Trans. Antennas and Propagation, Vol. 52, no. 3, pp. 329-342, 1997.

[4] R.M. Sharpe, J.B. Grant, N.J. Champagne, W.A. Johnson, R.E. Jorgenson, D.R. Wilton, W.J. Brown, J.W. Rockway, "EIGER: Electromagnetic Interactions 
GEneRalized", Antennas and Propagation Society International Symposium, 1997. IEEE., 1997 Digest, Vol. 4 , 13-18 July 1997, pp. 2366 - 2369.

Matrix Conditioning for Electrostatics

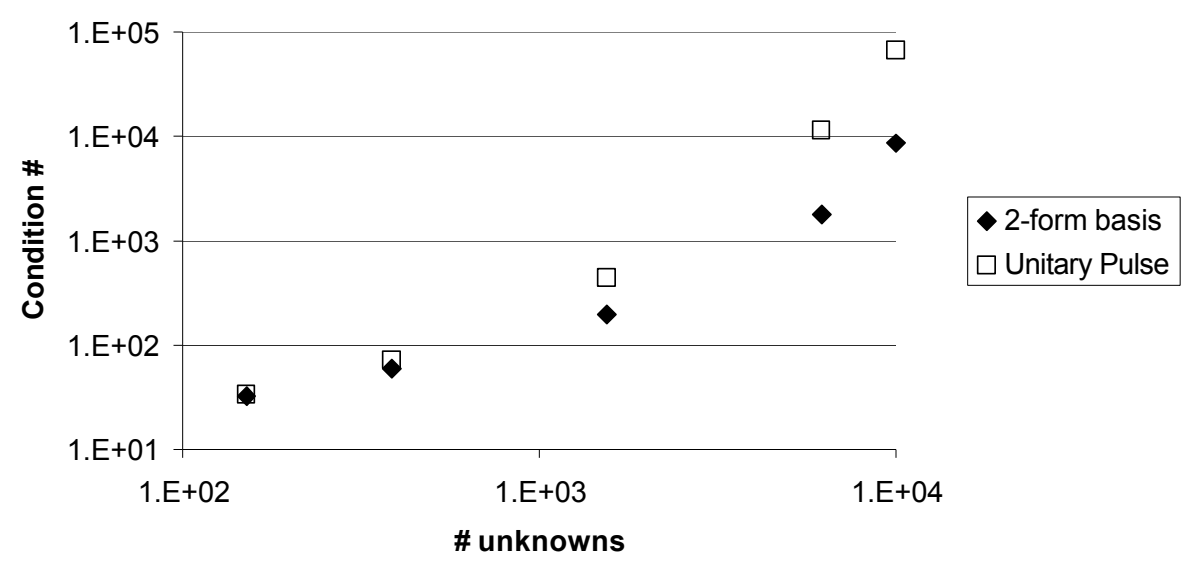

Figure 1. Matrix conditioning for a unit cube in free space at $1 \mathrm{~V}$.
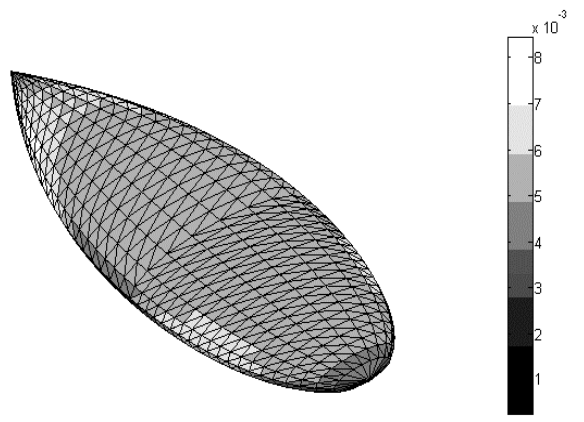

Figure 2. Magnitude of the surface currents on the NASA almond.

\section{Matrix Conditioning for EFIE}

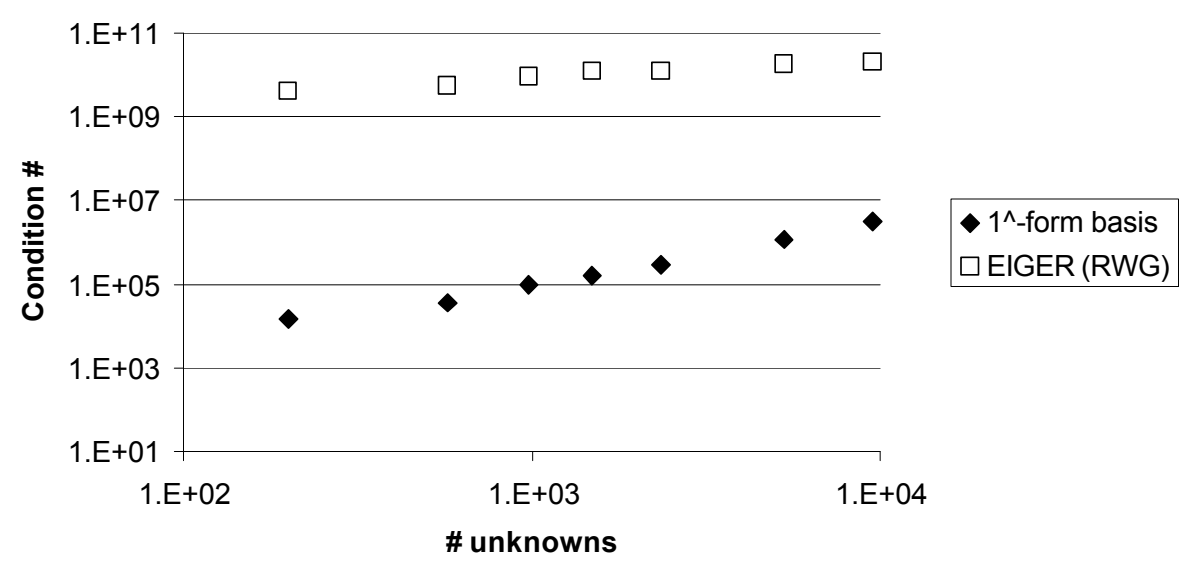

Figure 3. Matrix conditioning for the NASA almond. 